\title{
Salidroside attenuates hypoxia-induced pulmonary arterial smooth muscle cell proliferation and apoptosis resistance by upregulating autophagy through the AMPK-mTOR-ULK1 pathway
}

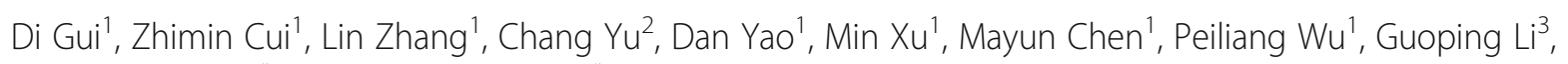
Liangxing Wang ${ }^{1 *}$ and Xiaoying Huang ${ }^{1 *}$

\begin{abstract}
Background: Recent studies have shown that both adenosine monophosphate activated protein kinase (AMPK) and the mammalian target of rapamycin (mTOR) are energy sensors and are related to autophagy. Our recent reports have shown that salidroside can exert protective effects against hypoxia-induced pulmonary arterial smooth muscle cell (PASMC) proliferation and apoptosis resistance through the AMPK pathway. This study aims to explore the relationship among AMPK, mTOR and ULK1 in PASMCs under hypoxic conditions and to investigate whether the protective effects of salidroside are related to the autophagic cell death pathway.

Methods: Rat PASMCs were cultured and divided into five groups: the normoxia, hypoxia, hypoxia + MHY1485 (mTOR agonist), hypoxia + rapamycin (mTOR inhibitor) and hypoxia + salidroside groups. Hypoxic cells were treated as indicated for $24 \mathrm{~h}$. Cell viability was evaluated by the CCK-8 assay. Cell apoptosis was measured by the TUNEL assay. The autophagy flux of PASMCs was evaluated with tandem mRFP-GFP fluorescence microscopy. Autophagosomes were detected by electron microscopy. Protein expression of LC3, p62, AMPK, P-AMPK (Thr 172), P-ULK1 (Ser 555 and Ser 317), mTOR, P-mTOR (Ser 2448), ULK1 and P-ULK1 (Ser 757) was detected by western blot assay.

(Continued on next page)
\end{abstract}

\footnotetext{
* Correspondence: wzyxywlx@163.com; zjwzhxy@126.com

'Division of Pulmonary Medicine, The First Affiliated Hospital of Wenzhou Medical University, Key Laboratory of Heart and Lung, Wenzhou, Zhejiang 325000, People's Republic of China

Full list of author information is available at the end of the article
} 
(Continued from previous page)

Results: PASMC proliferation and apoptosis resistance were observed under hypoxic conditions. Autophagy flux, the number of autophagosomes and the LC3II/LC3I ratio were increased in the hypoxia group compared with the normoxia group, whereas p62 expression was decreased. Treatment with rapamycin or salidroside reversed hypoxia-induced PASMC proliferation and apoptosis resistance and further increased autophagy flux, autophagosome levels and the LC3II/LC3I ratio but decreased p62 expression. Treatment with MHY1485 reversed hypoxia-induced PASMC apoptosis resistance and decreased autophagy flux as well as increased autophagosome levels, the LC3II/LC3I ratio and p62 expression. P-AMPK (Thr 172) and P-ULK1 (Ser 555) of the AMPK-ULK1 pathway were increased in the hypoxia group and were further increased in the salidroside group. Rapamycin and MHY1485 had no effect on either P-AMPK (Thr 172) or P-ULK1 (Ser 555). Phosphorylation of ULK1 at serine 317 did not significantly affect the five groups. Furthermore, P-mTOR (Ser 2448) and P-ULK1 (Ser 757) of the AMPK-mTOR-ULK1 pathway were decreased in the hypoxia group and were further decreased in the salidroside group. MHY1485 increased the expression of both P-mTOR(Ser 2448) and P-ULK1(Ser 757), whereas rapamycin had the opposite effect.

Conclusions: Salidroside might inhibit hypoxia-induced PASMC proliferation and reverse apoptosis resistance via the upregulation of autophagy through both the AMPKa1-ULK1 and AMPKa1-mTOR-ULK1 pathways.

Keywords: PASMCs, Hypoxia, Ampk, mTOR, ULK1, Autophagy

\section{Background}

The pathogenesis of pulmonary hypertension $(\mathrm{PH})$ consists of intima lesions, medial vascular remodeling and adventitial remodeling [1]. Pulmonary arterial smooth muscle cells (PASMCs) are the main constituents of medial layer of vessels. Recent studies have revealed that in patients with $\mathrm{PH}$, PASMC hyperplasia plays a key role in medial vascular thickening [2], and their resistance to apoptosis also determines the ultimate fate of PASMCs in PH [1].

Adenosine monophosphate activated protein kinase (AMPK) is an enzyme that can be activated by changes in the AMP/ATP ratio. It can maintain the balance of energy metabolism [3]. AMPK is a heterotrimer that consists of $\alpha, \beta$ and $\gamma$ subunits. Both $\beta$ and $\gamma$ subunits are regulatory subunits, but the $\alpha$ subunit is a catalytic subunit. The phosphorylation of $\alpha$ subunit at the threonine 172 residue is an essential step for the activation of AMPK [4]. In a variety of diseases, AMPK plays a protective role. It has been reported that metformin inhibits endothelin-1-induced PASMC proliferation via AMPK activation [5]. Additionally, our previous studies have shown that the selective AMPK activator 5 '-ami-noimidazole-4-carboxamide ribonucleoside (AICAR) can exert protective effects against hypoxia-induced PASMC proliferation and apoptosis resistance [6, 7]. Therefore, AMPK plays an important role in inhibiting hypoxic pulmonary hypertension (HPH).

Mammalian target of rapamycin (mTOR) is a serine/ threonine protein kinase and is also an energy sensor. It is important in the proliferation of PASMCs. Xueping Liu et al. [8] have revealed that mTOR siRNA could inhibit the proliferation of PASMCs under hypoxic conditions. Additionally, Vera P. Krymskaya et al. [9] have revealed that rapamycin, which inhibits mTORC1 signaling, could inhibit chronic hypoxia-induced rat PASMC proliferation.

As energy sensors, AMPK and mTOR have a close relationship. AMPK can downregulate mTOR expression through different pathways $[10,11]$. It has been reported that aspirin can help protect against the development of colorectal cancer through the activation of autophagy via upregulating AMPK activity and downregulating mTOR activity [12]. It was reported that the activation of autophagy through the modulation of mTOR-related signaling could result in pro-apoptotic effects on gastric cancer cells [13]. Additionally, in the research of idiopathic pulmonary fibrosis (IPF), Yair Romero et al. [14] found that the persistent activation of mTOR-related pathways decreased the activation of autophagy, which contributed to apoptosis resistance in the IPF fibroblasts. Hypoxia-induced PASMC proliferation possesses several characteristics that are similar to tumor cells [15]. The present study aimed to identify if autophagy might be regulated by the AMPK-mTOR pathway and to confirm if the stimulation of autophagy might exert a protective effect on $\mathrm{HPH}$.

Salidroside has many biological properties, such as cardioprotective, anti-cancer, anti-fatigue and antiviral effects [16-19]. Our previous reports have suggested that salidroside can exert protective effects against hypoxia-induced PASMC hyperproliferation and apoptosis resistance through an AMPK $\alpha 1$-dependent pathway [7]. However, the downstream signaling pathway remains unclear. In this study, we aimed to investigate the downstream signaling pathway of AMPK and reveal whether autophagy plays an essential role in hypoxiainduced PASMC proliferation and apoptosis resistance; additionally, we sought to investigate whether salidroside 
could attenuate $\mathrm{HPH}$ by regulating autophagy through the AMPK-mTOR pathway.

\section{Methods \\ Reagents}

Salidroside, 4,6-dimorpholino-N-(4-nitrophenyl)-1,3,5-triazin-2-amine (MHY1485) and collagenase type I were obtained from Sigma (St Louis, MO, USA). Rapamycin was obtained from LC laboratories (Woburn, MA, USA). Dulbecco's modified Eagle medium (DMEM, high glucose), streptomycin, penicillin $\mathrm{G}$ and fetal bovine serums (FBS) were obtained from Gibco BRL (Gaithersburg, MD, USA). The rabbit antibodies against LC3B, GAPDH, P-ULK1 (Ser 757 and Ser 317), mTOR, P-mTOR (Ser 2448) and p62 were obtained from Cell Signaling Technology (Beverly, MA, USA). The rabbit antibody against P-ULK1 (Ser 555) was obtained from US Biological (Swampscott, MA, USA). The rabbit antibody against P-AMPK (Thr 172) was obtained from Abways Technology (Shanghai, China). The rabbit antibody against AMPK was obtained from Abcam (Cambridge, UK). Cell counting kit-8 (CCK-8) was purchased from Dojindo Laboratories (Kumamoto, Japan). The in-situ Cell Death Detection Kit was purchased from Roche Diagnostics (Penzberg, Germany). Tandem monomeric RFP-GFP-tagged LC3 (tfLC3) was purchased from Genechem (Shanghai, China).

\section{Cell culture and treatment}

Rat PASMCs were derived from pulmonary arteries as described previously $[6,7]$ and were cultured in DMEM supplemented with $100 \mu \mathrm{g} / \mathrm{ml}$ streptomycin, $100 \mathrm{IU} / \mathrm{ml}$ penicillin and $10 \% \mathrm{FBS}$. Then, cells were divided into five groups: the normoxia $(\mathrm{N})$, hypoxia $(\mathrm{H})$, hypoxia $+\mathrm{MHY} 1485$ (mTOR agonist, $2 \mu \mathrm{mol} / \mathrm{L}$ ), hypoxia + rapamycin (mTOR inhibitor, $0.5 \mu \mathrm{mol} / \mathrm{L})$, hypoxia + salidroside $(499.5 \mu \mathrm{mol} / \mathrm{L})$ groups. Hypoxia-treated PASMCs were treated as indicated for $24 \mathrm{~h}$. All hypoxia groups were kept for $24 \mathrm{~h}$ in the hypoxia incubator at $37{ }^{\circ} \mathrm{C}$ with $5 \% \mathrm{CO}_{2}, 5 \% \mathrm{O}_{2}$ and $90 \% \mathrm{~N}_{2}$, whereas the normoxia group was kept in a normal incubator at $37{ }^{\circ} \mathrm{C}$ with $21 \% \mathrm{O}_{2}, 5 \% \mathrm{CO}_{2}$ and $74 \% \mathrm{~N}_{2}$.

\section{Cell viability assay}

Cell viability was determined by the CCK- 8 assay. PASMCs were seeded in 96-well microplates at a concentration of $1 \times 10^{4}$ cells/well. After they were preincubated in complete medium at $37{ }^{\circ} \mathrm{C}$ in $21 \% \mathrm{O}_{2}$ and $5 \% \mathrm{CO}_{2}$ for $24 \mathrm{~h}$, PASMCs were pretreated with salidroside, rapamycin or MHY1485 before exposure to hypoxia. Cell growth was observed under a microscope before the CCK- 8 assay. After $24 \mathrm{~h}$ of hypoxia, CCK- 8 was added to the cells at a concentration of $10 \mu \mathrm{l} /$ well for $2 \mathrm{~h}$. A microplate reader was used to determine the absorbance at $450 \mathrm{~nm}$.

\section{Cell apoptosis detection}

After pretreatment, cells that adhered to the cover slips were fixed with fresh $4 \%$ paraformaldehyde for $1 \mathrm{~h}$ at $15{ }^{\circ} \mathrm{C}-25{ }^{\circ} \mathrm{C}$. Cells were incubated with $3 \% \mathrm{H}_{2} \mathrm{O}_{2}$ for 10 min at $15{ }^{\circ} \mathrm{C}-25{ }^{\circ} \mathrm{C}$. Then, $0.1 \%$ Triton $\mathrm{X}-100$ was incubated with the cells for $15 \mathrm{~min}$ at room temperature. Both anti-goat serum and 5\% BSA were used to block non-specific binding. An in-situ Cell Death Detection Kit was used according to the manufacturer's instructions. DAB and hematoxylin were used for cell staining. Light microscopy was used to observe the cover slips. Five randomly selected fields from each cover slip were analyzed to determine the percentage of terminal deoxynucleotidyl (TUNEL)-positive cells.

\section{Autophagy flux detected by tandem mRFP-GFP fluorescence microscopy}

Cells were seeded onto 6-well microplates at a concentration of $5 \times 10^{4}$ cells/well. After preincubation in complete medium at $37{ }^{\circ} \mathrm{C}$ in $21 \% \mathrm{O}_{2}$ and $5 \% \mathrm{CO}_{2}$ for $24 \mathrm{~h}$, the cells were transfected with tfLC3 according to the manufacturer's instructions to monitor autophagy flux. Eight hours after transfection, the cells were washed with PBS; next, complete culture medium was added to the cells. At $48 \mathrm{~h}$ after transfection, the cells were treated with salidroside, rapamycin or MHY1485. Cells were incubated in the hypoxia chamber for another $24 \mathrm{~h}$. Finally, the samples were examined under a fluorescence microscope (Nikon, Tokyo, Japan).

\section{Autophagosome detection of rat PASMCs}

Cells were fixed with both $2.5 \%$ glutaraldehyde and $1 \%$ osmic acid in sequence and then stained with $1 \%$ uranyl acetate. Acetone was used to dehydrate the cells, and then the cells were embedded in epoxy resin 812 . Ultramicrotome slices V (Sweden) were used to cut the fixed cells into ultrathin sections. The number of autophagosomes in the cells was evaluated with Hitachi H-600 transmission electron microscopy (Hitachi, Japan).

\section{Detection of autophagy-related proteins LC3B and p62 by western blotting analysis}

Cells were harvested and incubated with ice-cold RIPA buffer containing PMSF. Then, the samples were centrifuged at $4{ }^{\circ} \mathrm{C}$ at $12000 \mathrm{rpm}$ for $15 \mathrm{~min}$. The Bradford method was used to quantify the protein concentrations. A total of $40 \mu \mathrm{g}$ of protein from each group was separated using $12 \%$ SDS-PAGE. The proteins were transferred to PVDF membranes and blocked with 5\% skimmed milk. Specific primary antibodies against LC3B $(1: 1000,2775 S)$ and p62 $(1: 1000,5114 S)$ were used to detect the proteins. GAPDH $(1: 1000,5174 S)$ was used as an internal control. Horseradish peroxidase-conjugated 
secondary antibodies were incubated with the proteins at a 1:10,000 dilution. Quantity one-4.6.2 software (BioRad Laboratories, Hercules, CA, USA) was used to quantify the density of immunoblots after the detection of immunoreactive bands with BeyoECL Plus reagents (Beyotime, China).

The AMPK-ULK1 pathway-related proteins AMPKa1, P-AMPKa1 (Thr 172), P-ULK1 (ser 555) and P-ULK1 (ser 317 ) were detected by western blotting analysis A total of $40 \mu \mathrm{g}$ of protein from each group was separated using $8 \%$ SDS-PAGE. Specific primary antibodies against AMPK $\alpha 1$ (1:2000, ab32047), phosphorylated AMPK $\alpha 1$ (1:2000, CY5556), phosphorylated ULK1 (Ser 555, 1:1000, U1500-70F) and phosphorylated ULK1 (Ser 317, 1:1000, 12753S) were used to detect the proteins. The experimental procedures for western blotting analysis were performed as described above.
The mTOR-ULK1 pathway-related proteins $\mathrm{mTOR}$, P-mTOR, ULK1 and P-ULK1 (ser 757) were detected by western blotting analysis

A total of $40 \mu \mathrm{g}$ of protein from each group was separated using $8 \%$ SDS-PAGE. Specific primary antibodies against mTOR (1:1000, 2983S), phosphorylated mTOR (1:1000, 5536S), ULK1 (1:1000, $8054 \mathrm{~s})$ and phosphorylated ULK1 (Ser 757, 1:1000, 14202S) were used to detect the proteins. The experimental procedures for western blotting analysis were performed as described above.

\section{Statistical analysis}

The results were expressed as the mean \pm standard deviation (SD). Statistical significance was determined with one-way ANOVA followed by the least significant difference (LSD) test. A value of $P<0.05$ was considered to be statistically significant. All calculations were performed by SPSS version 21.0.

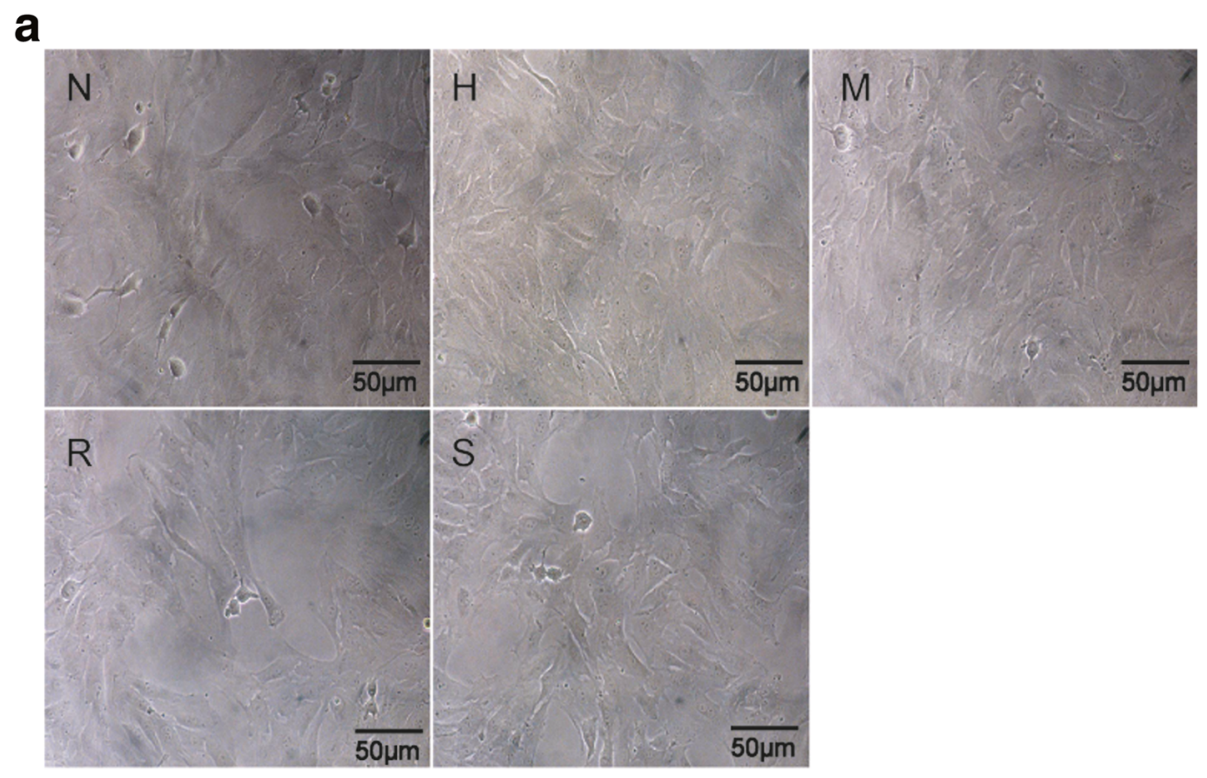

b

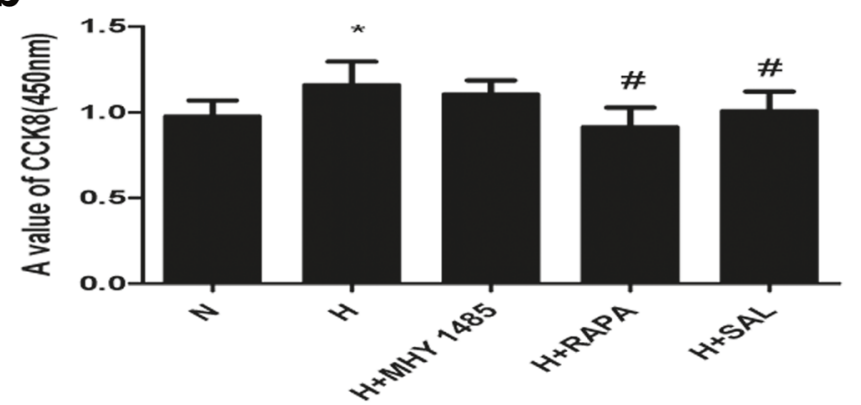

Fig. 1 Autophagy is involved in hypoxia-induced PASMC proliferation. a Cell density in each group as viewed under a microscope. b CCK-8 values in each group. ${ }^{*} p<0.05$ vs. the $\mathrm{N}$ group; and $\# p<0.05$ vs. the $\mathrm{H}$ group 


\section{Results}

Autophagy was involved in hypoxia-induced PASMC proliferation and apoptosis resistance

PASMCs were treated with hypoxia with or without rapamycin or MHY1485 to demonstrate the role of autophagy in hypoxia-induced PASMC proliferation and apoptosis resistance. As shown in Fig. 1a and b, the density of PASMCs under the microscope in the $\mathrm{H}$ group was greater than that in the $\mathrm{N}$ group. Additionally, the viability of hypoxiatreated PASMCs was obviously increased $(P<0.05)$. The density and viability of cells treated with rapamycin were reduced $(P<0.05)$, whereas MHY1485 had no effect on cell density and viability $(P>0.05)$. As shown in Fig. $2 \mathrm{a}$ and $\mathrm{b}$, the apoptosis index of PASMCs under hypoxic conditions was obviously decreased $(P<0.05)$. The apoptosis index of PASMCs treated with rapamycin or MHY1485 was increased $(P<0.05)$. Meanwhile, the number of autophagosomes, the ratio of LC3II to LC3I and autophagic flux were increased and the expression of p62 was decreased in cells treated with hypoxia. These changes were further enhanced in PASMCs treated with rapamycin (Figs. 3, 4 and 5).
Therefore, we infer that hypoxia could enhance autophagy, which can further be enhanced by rapamycin. In PASMCs treated with MHY1485, although the number of autophagosomes and the ratio of LC3II to LC3I were increased, the autophagic flux of PASMCs was decreased, and the expression of p62 was increased (Figs. 3, 4 and 5). Therefore, we concluded that MHY1485 could inhibit autophagy. These results indicate that the increase in autophagy under hypoxic conditions might compensatory, and further enhanced autophagy may decrease PASMC proliferation and increase PASMCs apoptosis. The detailed mechanisms underlying the decrease in autophagy, increase in the apoptosis index, and lack of effects on proliferation following MHY1485 treatment in hypoxic PASMCs were investigated next.

\section{Enhanced autophagy flux may underlie the effects of salidroside on PASMC proliferation and apoptosis resistance under hypoxic conditions}

To confirm autophagy activation following different interventions, we transfected tfLC3 into PASMCs (Fig. 3). Since the GFP signal is sensitive to acidic

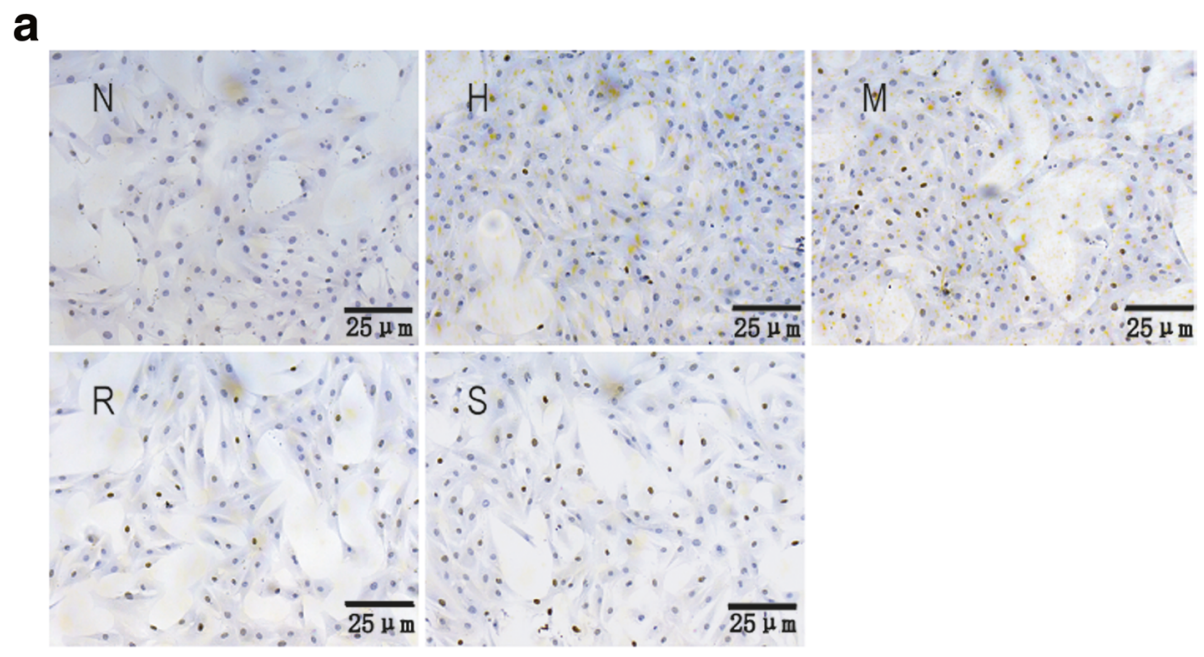

b

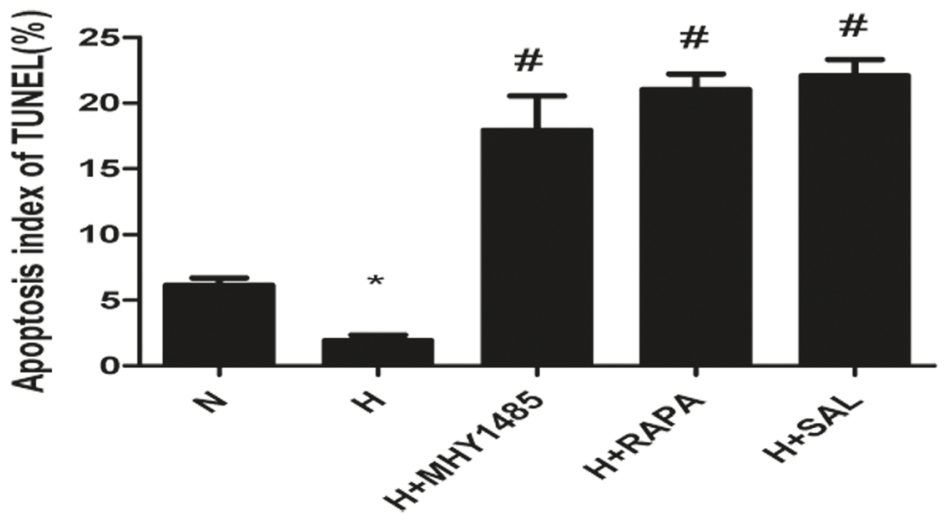

Fig. 2 Autophagy is involved in hypoxia-induced PASMC apoptosis resistance. a Images of cell apoptosis in each group as determined by the TUNEL assay. $\mathbf{b}$ Quantitative analysis of the cell apoptosis index by TUNEL assay. ${ }^{*} p<0.05$ vs. the N group; and \#p $<0.05$ vs. the H group 

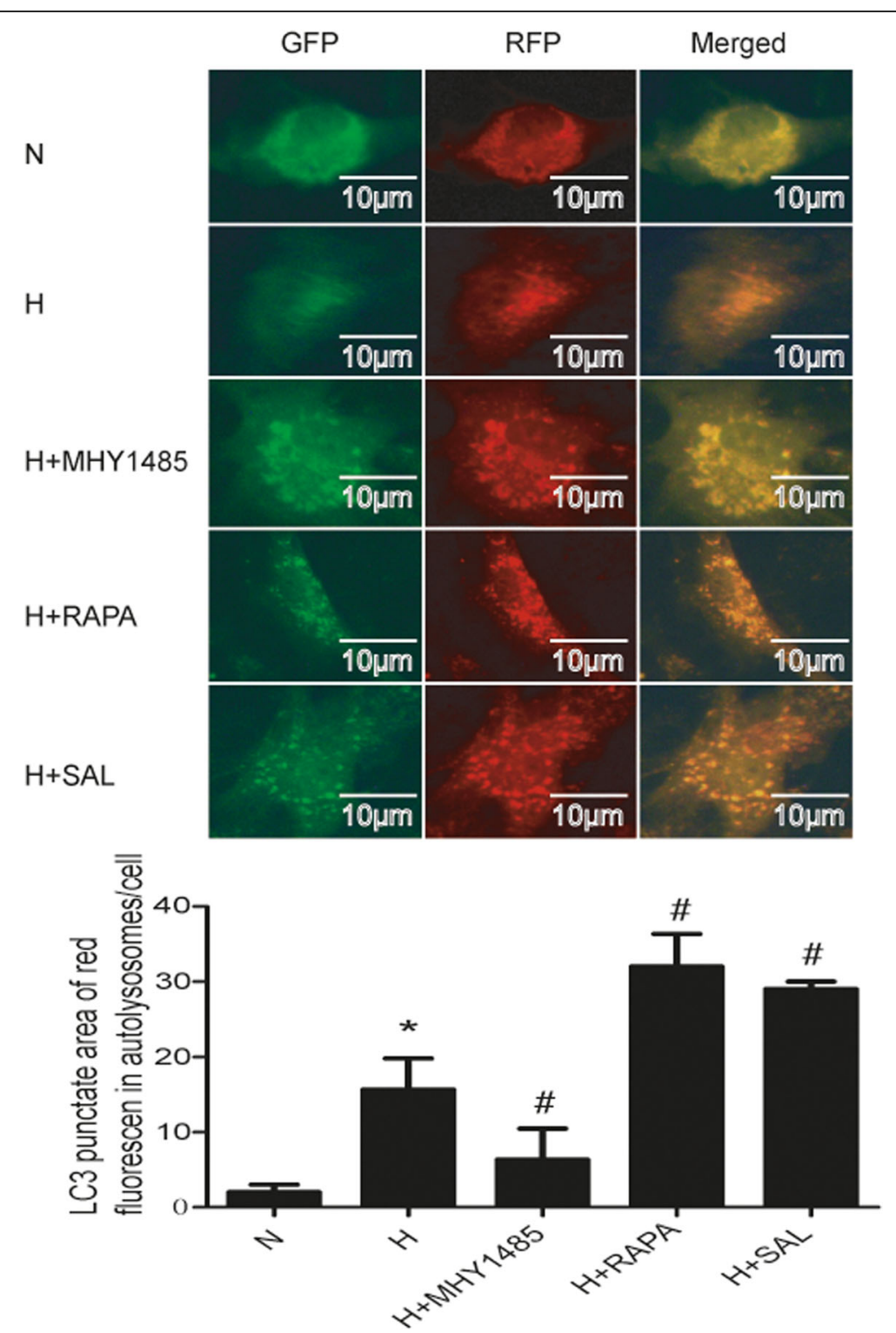

Fig. 3 Enhanced autophagy flux may be involved in the effects of salidroside on PASMC proliferation and apoptosis resistance under hypoxic conditions. After transfection with tfLC3, PASMCs were exposed to hypoxia and treated with MHY1485, rapamycin or salidroside; next, the cells were observed under a fluorescence microscope. ${ }^{*} p<0.05$ vs. the $\mathrm{N}$ group; and $\# p<0.05$ vs. the $\mathrm{H}$ group

conditions, this form of LC3 displays only red fluorescence when located in autolysosomes. Increased red spots were observed in hypoxic cells. Furthermore, an increased number of red spots were observed in cells treated with rapamycin and salidroside. However, both green and red spots were observed in cells treated with MHY1485. These data revealed that hypoxia enhanced the autophagy flux of cells, which was further enhanced by rapamycin and salidroside. However, MHY1485 weakened the autophagy flux of PASMCs.
Increased numbers of autophagosomes may be related to the salidroside-induced decrease in the proliferation and increase in the apoptosis of PASMCs under hypoxic conditions

To confirm the number of autophagosomes in PASMCs after different interventions, transmission electron microscopy was performed (Fig. 4). The number of autophagosomes were increased in cells under hypoxic conditions. The number of autophagosomes were further increased in cells treated with salidroside, rapamycin and MHY1485. These results together with the 

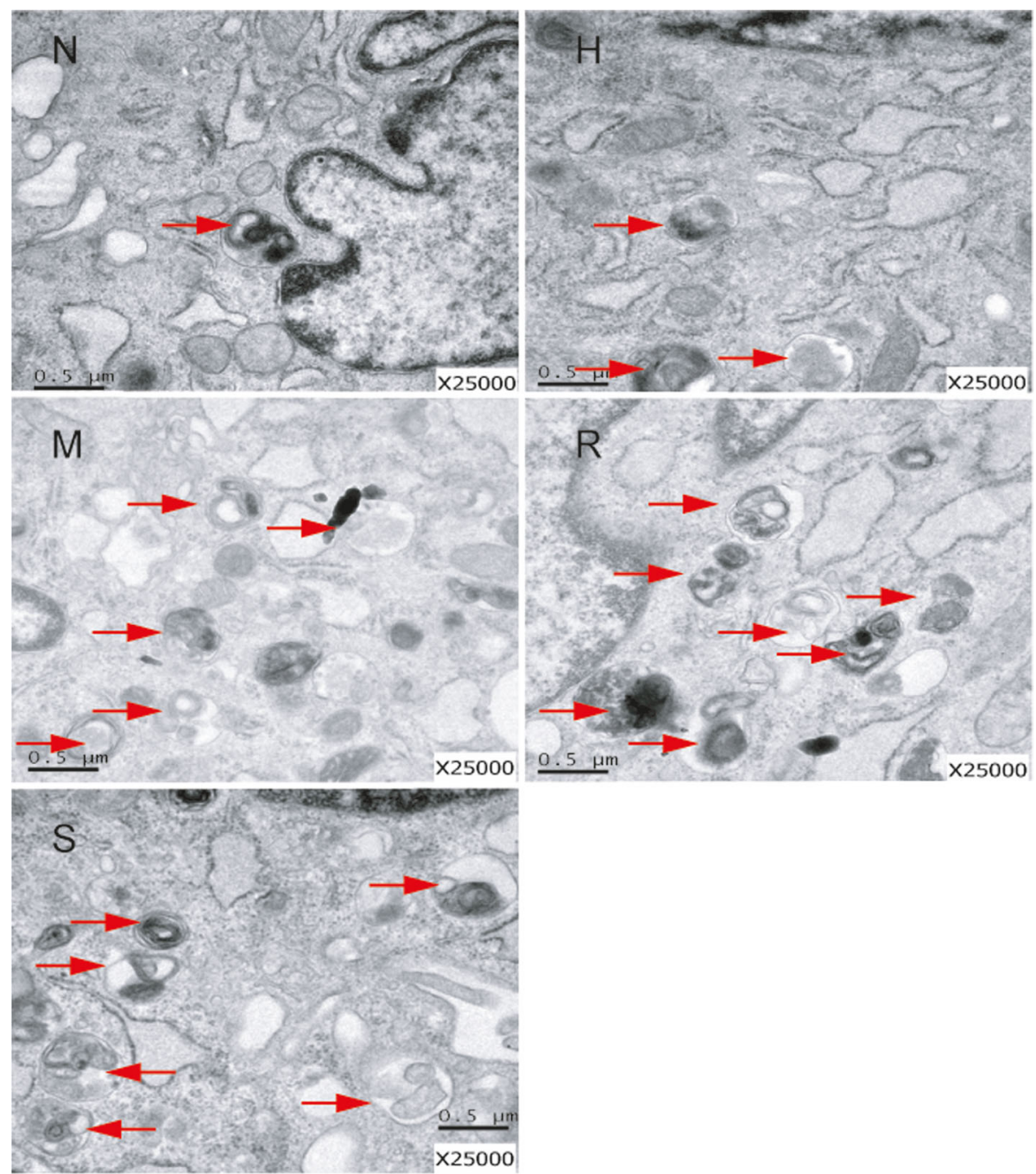

Fig. 4 Increased numbers of autophagosomes may be related to salidroside-induced decreases in the proliferation and increases in the apoptosis of PASMCs under hypoxic conditions. Images of autophagosomes in PASMCs of each group acquired by Hitachi H-600 transmission electron microscopy. The control group exhibited less autophagosomes than the hypoxia group. The salidroside group exhibit increased numbers of autophagosomes than the hypoxia group. Red arrow: autophagosomes

autophagy flux analysis indicated that salidroside increased the number of autophagosomes by activating autophagy. However, MHY1485 increased the number of autophagosomes through suppressing lysosomal fusion.

\section{Increased autophagy may be related to the} salidroside-induced attenuation of PASMC proliferation and apoptosis resistance under hypoxic conditions

To confirm autophagy activation following different interventions, protein levels of LC3 and p62 were detected by western blotting analysis (Fig. 5). The ratio of LC3II to LC3I was increased and p62 expression was diminished in the hypoxia group $(P<0.05)$. In both the rapamycin and salidroside groups, changes in the ratio of LC3II to LC3I and p62 expression were further enhanced $(P<0.05)$. However, both the ratio of LC3II to LC3I and p62 expression were increased in the MHY1485 group compared to the hypoxia group $(P<0.05)$. These data together with autophagy flux and autophagosome detection revealed that rapamycin and salidroside further increases autophagy compared to hypoxia, which reverses PASMC proliferation and apoptosis resistance (Figs. 1a, b and 2a, b). However, increased levels of autophagosomes in the MHY1485 group resulted in increased LC3II expression, which may be related to the reversal of PASMC proliferation and apoptosis resistance.

\section{The AMPK-ULK1 (ser 555) pathway, which regulates} autophagy, is upregulated by salidroside treatment in PASMCs

To confirm whether the increase in autophagy induced by salidroside is dependent on the regulation of the 

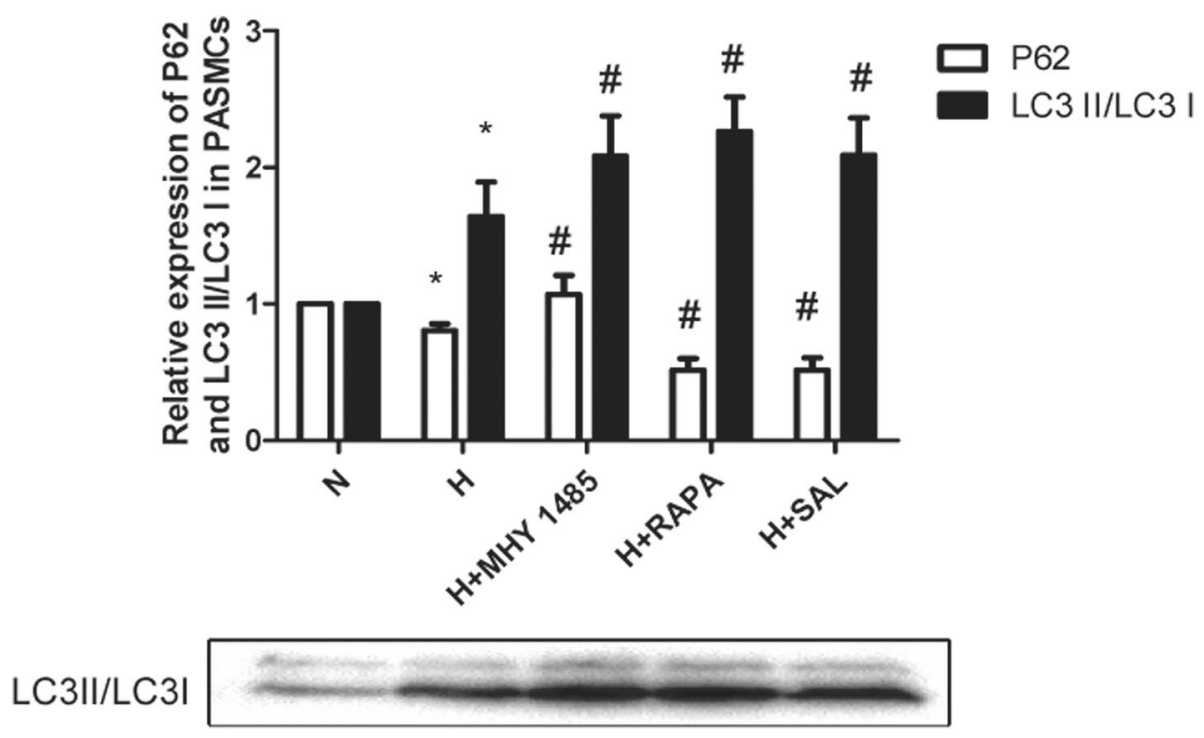

p62

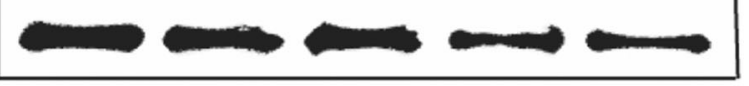

GAPDH

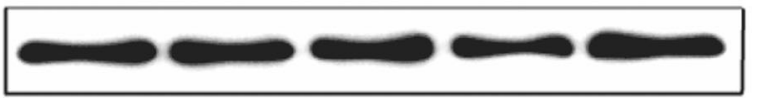

Fig. 5 Increased autophagy may be related to the salidroside-induced attenuation of PASMC proliferation and apoptosis resistance under hypoxic conditions. Images and quantitative analysis of p62 expression and the LC3II/LC3I ratio in PASMCs by western blot assay. GAPDH was used as an internal control. ${ }^{*} p<0.05$ vs. the $N$ group; and $\# p<0.05$ vs. the $H$ group

AMPK-ULK1 pathways, cells were treated with hypoxia and salidroside. The levels of AMPK, phosphorylated AMPK and phosphorylated ULK1 (Ser 555) were upregulated in the hypoxia group. The changes in AMPK, phosphorylated AMPK and phosphorylated ULK1 (Ser 555) expression were further enhanced in the salidroside group compared to the hypoxia group $(P<0.05)$. To further confirm whether the role of salidroside is mTOR dependent, cells were treated with the mTOR inhibitor rapamycin and the mTOR agonist MHY1485. The expression of these proteins in cells treated by MHY1485 and rapamycin was similar to that in the hypoxia group $(P>0.05)$. The phosphorylation of ULK1 at serine 317 had no significant effect on the five groups $(P>0.05)$ (Fig. 6a). These data revealed that salidroside can exert a protective effect through the AMPK-ULK1 (Ser 555) pathway (Fig. 7).

The mTOR-ULK1 (ser 757) pathway, which regulates autophagy, was downregulated by salidroside in PASMCs To confirm whether the increase in autophagy induced by salidroside was also dependent on the regulation of the mTOR-ULK1 pathway, mTOR, P-mTOR, ULK1 and P-ULK1 (S757) protein levels were detected (Fig. 6b). Protein expression of mTOR, phosphorylated mTOR and phosphorylated ULK1 (Ser 757) was decreased in the hypoxia group $(P<0.05)$. Changes in mTOR, phosphorylated mTOR and phosphorylated ULK1 (Ser 757) expression were further enhanced in the salidroside and rapamycin groups compared to the hypoxia group $(P<0.05)$. Protein expression levels of mTOR, phosphorylated mTOR and phosphorylated ULK1 (Ser 757) were increased in the MHY1485 group compared to the hypoxia group $(P<0.05)$. The expression of ULK1 contrasts that of P-ULK1 (Ser 757). These data revealed that salidroside can exert a protective effect through the mTOR-ULK1 (Ser 757) pathway (Fig. 7).

\section{Discussion}

Autophagy is the process by which cellular components are self-degraded. Portions of cytosol or organelles are sequestered by double-membrane autophagosomes, which fuse with lysosomes and are degraded by resident hydrolases [20]. Autophagy can be divided into three main types according to the different delivery pathways of cargo to the lysosome: macroautophagy, microautophagy and chaperone-mediated autophagy (CMA) [21]. Macroautophagy is the most widely studied process among the three main forms of autophagy, and the present study focuses on it. The process of autophagy consists of induction, vesicle nucleation, membrane elongation, autophagosome formation, autophagosome 


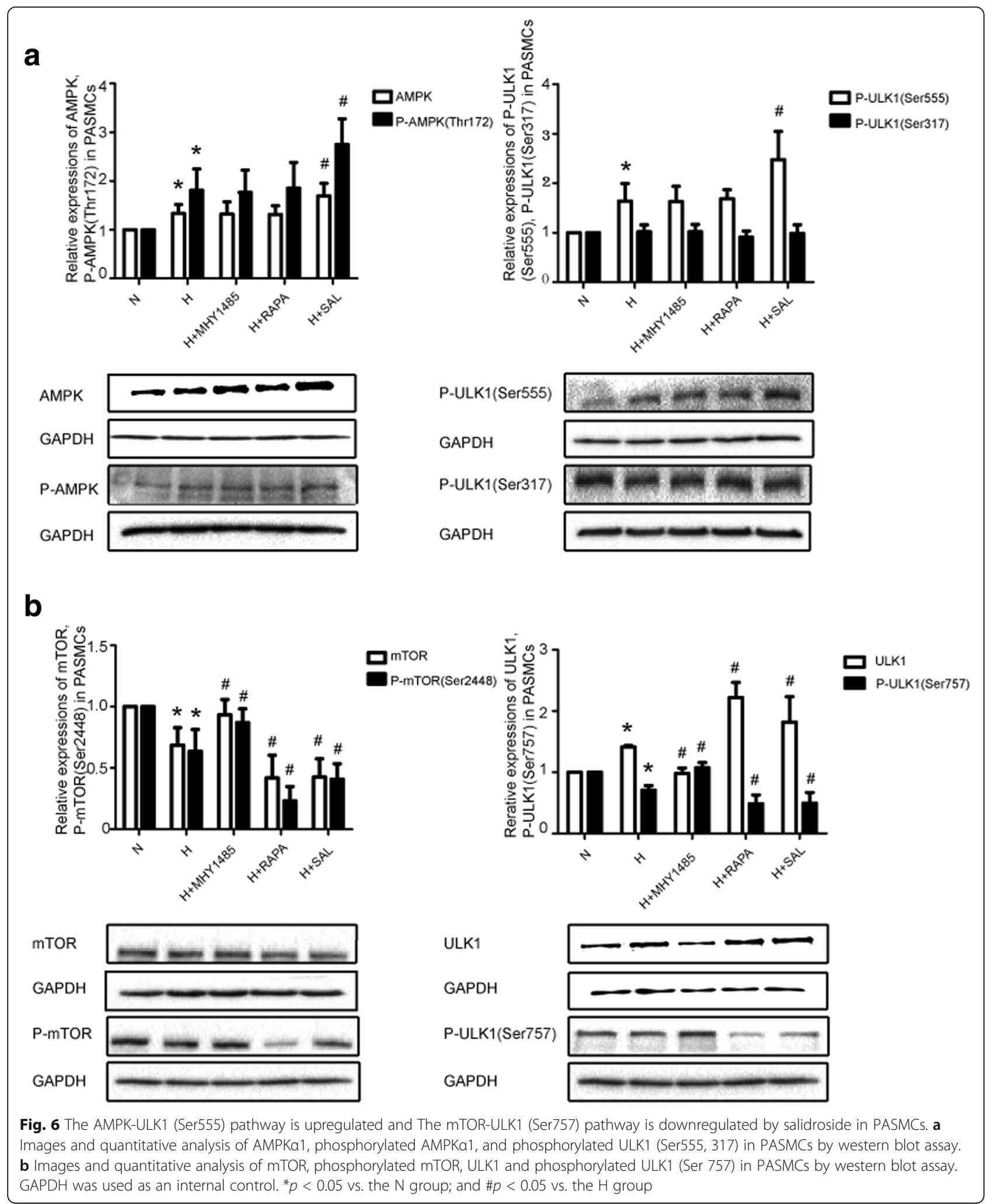

maturation/cargo assimilation, autophagosome lysosome fusion, autolysosomal acidification, substrate degradation and recycling [22]. Induction is initiated by the activation of the ATG1 complex [23]. UNC-51-like kinase 1 (ULK1) and 2 are the mammalian homologs of ATG1 [24]. Most importantly, ULK1 constantly associates with ATG13, and 


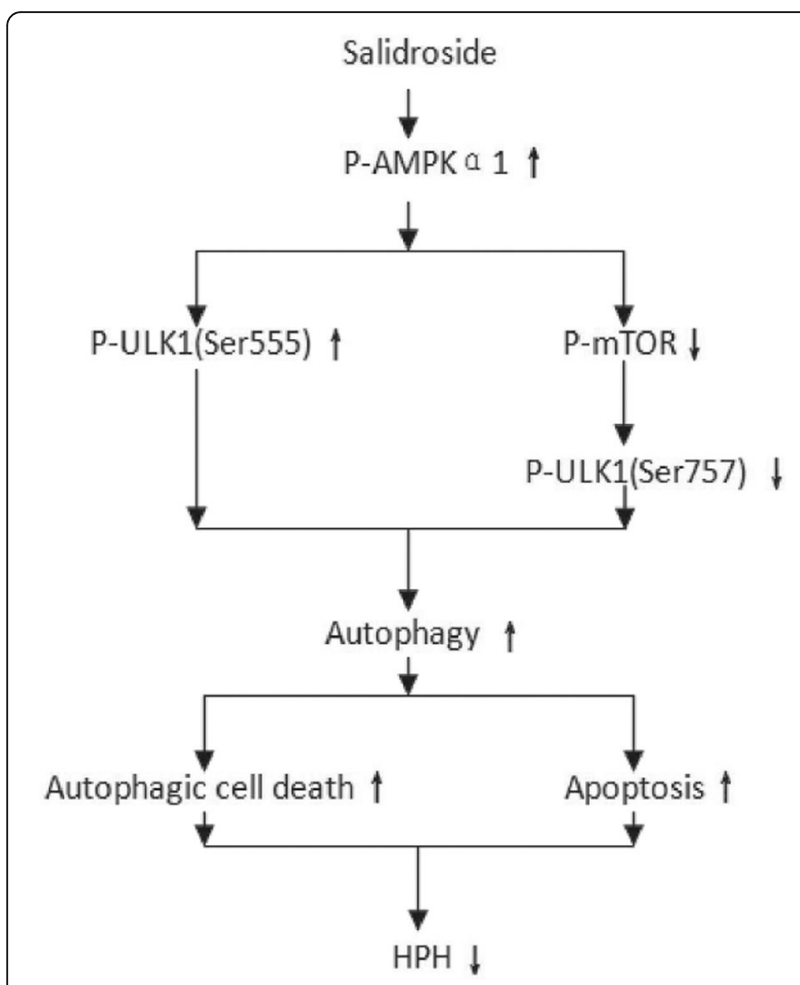

Fig. 7 The signaling pathways of this experiment. Salidroside exerted protective effects against hypoxic PASMCs via the upregulation of autophagy through both the AMPK-ULK1 (Ser 555) and AMPK-mTORULK1 (Ser 757) pathways

thus, ULK1 is the key regulatory protein for mammalian autophagy initiation [25].

$\mathrm{HPH}$ is one of the main complications of chronic obstructive pulmonary diseases, which is associated with pulmonary vascular remodeling caused by chronic hypoxia. Endothelial cell dysfunction, excessive contraction of blood vessels and smooth muscle cell hyperplasia are all associated with pulmonary vascular remodeling [26]. Therefore, inhibition of PASMC hyperproliferation and apoptosis resistance may be an efficient therapeutic strategy for $\mathrm{HPH}$.

In a previous study, we revealed that 5-Aminoimidazole4-carboxamide ribonucleotide (AICAR), an agonist of AMPK, decreases proliferation and increases the apoptosis index of hypoxic PASMCs [6]. Additional studies have revealed that salidroside has the same effect as AICAR [7]. However, its molecular mechanism has not been fully investigated.

The result of the CCK-8 assay showed that hypoxia increases the viability of PASMCs, and salidroside reverses the effect of hypoxia. Rapamycin, which enhances autophagy in cells, obviously inhibited the viability of PASMCs. As reported by Juliana Navarro-Yepes et al. [27], the autophagy-induced excessive degradation of essential cellular components may cause autophagic cell death. In the present study, MHY1485, an agonist of mTOR, slightly decreased the viability of PASMCs. Yeon Ja Choi et al. [28] revealed that MHY1485 not only inhibits autophagy by activating mTOR but also suppresses lysosomal fusion. In addition, Daniel J. Klionsky et al. [29] revealed that the reasons for the accumulation of autophagosomes include upregulating autophagy induction and downregulating autophagosome degradation. In our study, although MHY1485 increased autophagosome and LC3BII levels, it inhibited autophagy flux, which concurred with the findings of Yeon Ja Choi et al. [28]. Although decreased autophagy flux may be related to an increase in the proliferation of PASMCs, increased LC3B could exert opposite effects [30]. This explains why MHY1485 did not have an effect on PASMC viability under hypoxic conditions. Altogether, we concluded that autophagy may be increased as a compensatory response to hypoxia and that further enhancements in autophagy may lead to a decrease in cell proliferation.

Using the TUNEL assay, the present study showed that hypoxia decreased the apoptosis index of PASMCs. Salidroside obviously reversed the effect of hypoxia. Rapamycin, which induces autophagy, also increased the apoptosis index of PASMCs under hypoxic conditions. As reported by Jacob M. Gump et al. [31], enhanced autophagy might selectively promote Fas-mediated apoptotic cell death. MHY1485 also increased the apoptosis index of PASMCs under hypoxic conditions, which may also be attributed to its pharmacological action. MHY1485 increased autophagosome and LC3B levels in a manner that is similar to the induction of autophagy. Furthermore, Shi Chen [32] reported that the accumulation of autophagosomes may aggravate alveolar macrophage apoptosis. Therefore, we infer that enhanced autophagy may increase PASMC apoptosis in $\mathrm{HPH}$.

In a previous study [7], we demonstrated that salidroside can exert protective effects on PASMCs under hypoxic conditions through an AMPK-dependent pathway. Dmitry A.Goncharov et al. [33] revealed that AMPK could reverse PASMC proliferation by downregulating mTORC1 signaling. mTOR is an autophagy-associated protein, and it can inhibit autophagy through the phosphorylation of ULK1 [25, 34]. Moreover, previous studies have revealed that AMPK can enhance autophagy by directly phosphorylating the serine sites of ULK1, which is the protein responsible for autophagy induction [34, 35]. Therefore, AMPK can phosphorylate ULK1 through both direct and indirect pathways. Altogether, we infer that salidroside may exert protective effects on PASMCs under hypoxic conditions through both the AMPKULK1 and AMPK-mTOR-ULK1 pathways.

In this study, we first investigated the AMPK-ULK1 pathway. We applied MHY1485, an mTOR activator, or rapamycin, an mTOR inhibitor. Then, we determined if 
the changes in the level of phosphorylated ULK1 in the hypoxia and salidroside group were mTOR dependent or mTOR independent by comparing their results with that of the MHY1485- and rapamycin-treated groups. Joungmok Kim et al. [34] revealed that AMPK can phosphorylate ULK1 at serine 317 and serine 777 in HEK293 cells. Additionally, the study by Daniel F. Egan et al. [35] revealed that AMPK can phosphorylate ULK1 at the serine 555, serine 467, serine 627 and threonine 574 residues in HEK293T cells. Therefore, we choose to detect ULK1, a key protein of the mTOR independent pathway, at the serine 555 and serine 317 residues. The levels of AMPK, phosphorylated AMPK and phosphorylated ULK1 (Ser 555) were upregulated under hypoxic conditions, and salidroside further enhanced the changes in the levels of these proteins. However, the expression of these proteins in PASMCs treated with MHY1485 or rapamycin was similar to that in the hypoxia group. The phosphorylation of ULK1 at serine 317 did not lead to significant changes in the five groups. Therefore, we concluded that the AMPK-ULK1 (Ser 555) pathway is involved in the regulation of autophagy by salidroside in PASMCs. In a future study, we will further investigate other residues of ULK1 (Fig. 7).

The mTOR-ULK1 pathway was also investigated. Recent studies have revealed that AMPK can inhibit the activity of mTOR $[10,11]$. On the other hand, mTOR can inhibit autophagy through the phosphorylation of ULK1 at serine $757[25,34]$. In our study, the levels of mTOR, phosphorylated mTOR and phosphorylated ULK1 (Ser 757) were downregulated in the hypoxia group. Salidroside and rapamycin further enhanced the changes in the expression of these proteins, and MHY1485 had the opposite effect. The expression of total ULK1 is opposite of that of P-ULK1 (Ser 757). Therefore, we concluded that the mTOR-ULK1 (Ser757) pathway was also involved in the regulation of autophagy by salidroside in PASMCs (Fig. 7).

\section{Summary}

In our research, autophagy flux, autophagosome levels and the ratio of LC3II to LC3I were increased and p62 expression was decreased in PASMCs under hypoxic conditions; additionally, salidroside further enhanced the abovementioned changes and attenuated hypoxia-induced pulmonary arterial smooth muscle cell proliferation and apoptosis resistance. We concluded that the increase in autophagy in hypoxic PASMCs was a compensatory response and that salidroside could further enhance autophagy. Enhanced autophagy induced by salidroside through both the AMPKa1-ULK1 (Ser 555) and AMPK 1 1-mTOR-ULK1 (Ser 757) pathways could decrease cell proliferation and increase cell apoptosis in PASMCs under hypoxic conditions.

\section{Conclusions}

In conclusion, our study demonstrated that salidroside effectively inhibits PASMC proliferation and apoptosis resistance under hypoxic conditions by upregulating autophagy through both the AMPKa1-ULK1 (Ser 555) and AMPK 1 1-mTOR-ULK1 (Ser 757) pathways. This study provides novel evidence of the protective effects of salidroside against $\mathrm{HPH}$.

\section{Abbreviations \\ AMPK: adenosine monophosphate activated protein kinase; ATG: Autophagy- related gene; DMEM: Dulbecco's modified Eagle medium; HPH: hypoxic pulmonary hypertension; IPAH: idiopathic pulmonary arterial hypertension; IPF: idiopathic pulmonary fibrosis; mTOR: mammalian target of rapamycin; PASMCs: pulmonary arterial smooth muscle cells; $\mathrm{PH}$ : pulmonary hypertension; Ser: serine; tfLC3: tandem monomeric RFP-GFP-tagged LC3; Thr: threonine; TSC: tuberous sclerosis proteins; and raptor: regulatory- associated protein of mTOR; ULK: UNC-51-like kinase}

\section{Acknowledgements}

Not applicable.

\section{Funding}

This study was supported by the Chinese National Natural Science Foundation Grants (No. 81473406), the Natural Science Foundation of Zhejiang Province Grants (Y17H010028), and a project of the Health Department of Zhejiang Province of China (2016DTA005).

\section{Availability of data and materials}

The data and materials are stored in the Key Laboratory of Heart and Lung of Wenzhou Medical University and can be requested from the first author and corresponding author.

\section{Authors' contributions}

$\mathrm{XYH}$ and DG designed the experiment and drafted the manuscript. DG, ZMC, LZ, CY, PLW, MX, and GPL performed the experiments. MYC, DY and CY participated in the statistical analyses. LXW and GPL helped draft the manuscript. LXW participated in the study design and coordinated the research group. All authors have read and approved the final manuscript.

\section{Ethics approval}

All experimental protocols were in accordance with the Guide for the Care and Use of Laboratory Animals published by the US National Institute of Health and were approved by the Animal Ethics Committee of Wenzhou Medical University. Additionally, all animals were handled humanely during the study protocol and during euthanasia.

Consent for publication

Not applicable.

\section{Competing interests}

The authors declare that they have no competing interests.

\section{Publisher's Note}

Springer Nature remains neutral with regard to jurisdictional claims in published maps and institutional affiliations.

\section{Author details}

${ }^{1}$ Division of Pulmonary Medicine, The First Affiliated Hospital of Wenzhou Medical University, Key Laboratory of Heart and Lung, Wenzhou, Zhejiang 325000, People's Republic of China. 'Department of Invasive Technology, The First Affiliated Hospital of Wenzhou Medical University, Wenzhou, Zhejiang 325000, People's Republic of China. ${ }^{3}$ Department of Respiratory Medicine, Tongde Hospital of Zhejiang Province, Hangzhou, Zhejiang 310013, People's Republic of China. 
Received: 16 May 2017 Accepted: 6 October 2017

Published online: 12 December 2017

\section{References}

1. Tuder RM, Marecki JC, Richter A, Fijalkowska I, Flores S, et al. Pathology of pulmonary hypertension. Clin Chest Med. 2007;28(1):23-vii.

2. Dorfmuller P. Pulmonary hypertension: pathology. Handb Exp Pharmacol. 2013;218:59-75.

3. Ruderman NB, Carling D, Prentki M, Cacicedo JM. AMPK, insulin resistance, and the metabolic syndrome. J Clin Invest. 2013;123(7):2764-72.

4. Viollet B, Horman S, Leclerc J, Lantier L, Foretz M, Billaud M, et al. AMPK inhibition in health and disease. Crit Rev Biochem Mol. 2010;45(4):276-95.

5. Wu YY, Liu L, Zhang YH, Wang GZ, Han D, Ke R, et al. Activation of AMPK inhibits pulmonary arterial smooth muscle cells proliferation. Exp Lung Res. 2014:40(5):251-8.

6. Huang XY, Fan R, Lu YY, Yu C, Xu XM, Zhang X, et al. Regulatory effect of AMP-activated protein kinase on pulmonary hypertension induced by chronic hypoxia in rats: in vivo and in vitro studies. Mol Biol Rep. 2014:41(6): 4031-41.

7. Chen MY, Cai H, Yu C, Wu PL, Xu XM, Fan R, et al. Salidroside exerts protective effects against chronic hypoxia-induced pulmonary arterial hypertension via AMPKa1-dependent pathways. Am J Transl Res. 2016;8(1):12-27.

8. Liu XP, Wang GS, You ZC, Qian P, Chen HP, Dou Y, et al. Inhibition of hypoxiainduced proliferation of pulmonary arterial smooth muscle cells by a mTOR siRNA-loaded cyclodextrin nanovector. Biomaterials. 2014;35:4401-16.

9. Krymskaya VP, Snow J, Cesarone G, Khavin I, Goncharov DA, Lim PN, et al. mTOR is required for pulmonary arterial vascular smooth muscle cell proliferation under chronic hypoxia. FASEB J. 2011;25(6):1922-33.

10. Lnoki K, Zhu TQ, Guan KL. TSC2 mediates cellular energy response to control cell growth and survival. Cell. 2003;115(5):577-90.

11. Gwinn DM, Shackelford DB, Egan DF, Mihaylova MM, Mery A, Vasquez DS, et al. AMPK phosphorylation of raptor mediates a metabolic checkpoint. Mol Cell. 2008:30(2):214-26.

12. Din FVN, Valanciute A, Houde VP, Zibrova D, Green KA, Sakamoto K, et al. Aspirin inhibits MTOR signaling, activates AMP-activated protein kinase, and induces autophagy in colorectal cancer cells. Gastroenterology. 2012;142(7):26-33.

13. Wang K, Liu R, Li JY, Mao JL, Lei YL, Wu JH, et al. Quercetin induces protective autophagy in gastric cancer cells: involvement of Akt-mTOR-and hypoxia-induced factor 1a-mediated signaling. Autophagy. 2011;7(9):966-78.

14. Romero Y, Bueno M, Ramirez R, Alvarez D, Sembrat JC, Goncharova EA, et al. mTORC1 activation decreases autophagy in aging and idiopathic pulmonary fibrosis and contributes to apoptosis resistance in IPF fibroblasts. Aging Cell. 2016;15(6):1103-12

15. Sutendra G, Michelakis ED. The metabolic basis of pulmonary arterial hypertension. Cell Metab. 2014;19(4):558-73.

16. Wu TJ, Zhou HP, Jin ZX, Bi SH, Yang XL, Yi DH, et al. Cardioprotection of salidroside from ischemia/reperfusion injury by increasing $\mathrm{N}$-acetylglucosamine linkage to celluar proteins. Eur J Pharmacol. 2009;613(1-3):93-9.

17. Hu XL, Lin $S X, Y u D H$, Qiu SF, Zhang XQ, Mei RH. A preliminary study: the anti-proliferation effect of salidroside on different human cancer cell lines. Cell Biol Toxicol. 2010;26(6):499-507.

18. Wiegant FAC, Surinova S, Ytsma E, Makkinje ML, Wikman G, Post JA. Plant adaptogens increase lifespan and stress resistance in C. Elegans. Biogerontology. 2009;10(1):27-42.

19. Wang HB, Ding YY, Zhou J, Sun $X L$, Wang SW. The in vitro and in vivo antiviral effects of salidroside from Rhodiola Rosea L. against coxsackievirus B3. Phytomedicine. 2009;16(2-3):146-55.

20. He C, Klionsky DJ. Regulation mechanisms and signaling pathways of Autophagy. Annu Rev Genet. 2009:43(1):67-93.

21. Yang ZF, Klionsky DJ. Eaten alive: a history of macroautophagy. Nat Cell Biol. 2010;12(9):814-22.

22. Klionsky DJ. The molecular machinery of autophagy: unanswered questions. J Cell Sci. 2005;118(Pt 1):7-18

23. Chen $Y Q$, Klionsky DJ. The regulation of autophagy-unanswered questions. J Cell Sci. 2011;124(Pt 2):161-70.

24. Mizushima N. The role of the Atg1/ULK1 complex in autophagy regulation. Curr Opin Cell Biol. 2010;22(2):132-9.

25. Shang LB, Chen S, Du FH, Li S, Zhao LP, Wang XD. Nutrient starvation elicits an acute autophagic response mediated by Ulk1 dephosphorylation and its subsequent dissociation from AMPK. P Natl Acad Sci USA. 2011;108(12): 4788-93.

26. Budhiraja R, Tuder RM, Hassoun PM. Endothelial dysfunction in pulmonary hypertension. Circulation. 2004;109(2):159-65.

27. Yepes JN, Burns M, Anadhan A, Khalimonchuk O, Razo LM, Vega BQ, et al. Oxidative stress, redox signaling, and autophagy: cell death versus survival. Antioxid Redox Signal. 2014;21(1):66-85.

28. Choi YJ, Park YJ, Park JY, Jeong HO, Kim DH, Ha YM, et al. Inhibitory effect of mTOR activator MHY 1485 on autophagy: suppression of lysosomal fusion. PLoS One. 2012;7(8):e43418.

29. Klionsky DJ, Abdalla FC, Abeliovich H, Abraham RT, Arozena AA, Adeli K, et al. Guidelines for the use and interpretation of assays for monitoring autophagy. Autophagy. 2012:8(4):445-544.

30. Lee SJ, Smith A, Guo LP, Alastalo TP, Li ML, Sawada H, et al. Autophagic protein LC3B confers resistance against hypoxia-induced pulmonary hypertension. Am J Respir Crit Care Med. 2011;183(5):649-58.

31. Gump JM, Staskiewicz L, Morgan MJ, Bamberg A, Riches DWH, Thorburn A. Autophagy variation within a cell population determines cell fate via selective degradation of Fap-1. Nat Cell Biol. 2014;16(1):47-54.

32. Chen S, Yuan JX, Yao SQ, Jin YL, Chen G, Tian W, et al. Lipopolysaccharides may aggravate apoptosis through accumulation of autophagosomes in alveolar macrophages of human silicosis. Autophagy 2015; 11(12): 2346-2357.

33. Goncharov DA, Kudryashova TV, Ziai H, Stansbury Kl, Delisser H, Krymskaya VP, et al. mTORC2 coordinates pulmonary artery smooth muscle cell metabolism, proliferation and survival in pulmonary arterial hypertension. Circulation. 2014;129(8):864-74.

34. Kim J, Kundu M, Viollet B, Guan KL. AMPK and mTOR regulate autophagy through direct phosphorylation of Ulk1. Nat Cell Biol. 2011;13(2):132-41.

35. Egan DF, Shackelford DB, Mihaylova MM, Gelino SR, Kohnz RA, Mair W, et al. Phosphorylation of ULK1 (hATG1) by AMP-activated protein kinase connects energy sensing to mitophagy. Science. 2011;331(6010):456-61.

\section{Submit your next manuscript to BioMed Central and we will help you at every step:}

- We accept pre-submission inquiries

- Our selector tool helps you to find the most relevant journal

- We provide round the clock customer support

- Convenient online submission

- Thorough peer review

- Inclusion in PubMed and all major indexing services

- Maximum visibility for your research

Submit your manuscript at www.biomedcentral.com/submit 